\title{
Lattice study of color confinement mechanism in Coulomb gauge
}

\section{T. Saito*}

Research Center for Nuclear Physics, Osaka University, Ibarakisi, Osaka 567-0044, Japan

E-mail: tsaito@rcnp.osaka-u.ac.jp

\section{A. Nakamura}

Research Institute for Information Science and Education, Hiroshima University,

Higashi-Hiroshima 739-8521, Japan

E-mail: nakamuraeriise.hiroshima-u.ac.jp

\section{H. Toki, Y. Nakagawa}

Research Center for Nuclear Physics, Osaka University,

Ibarakisi, Osaka 567-0044, Japan

E-mail: toki@rcnp.osaka-u.ac.jp, nkgw@rcnp.osaka-u.ac.jp

We investigate the color-Coulomb confinement scenario using a SU(3) lattice gauge simulation. It is shown that the color-Coulomb heavy-quark potential, defined from the instantaneous part of the gluon propagator in Coulomb gauge, behaves as a linearly rising potential at large distances, and the resulting Coulomb string tension is greater than the Wilson-loop string tension. We study also the behavior of the color-Coulomb instantaneous part in the QGP phase. It is found that the linearity of the color-Coulomb instantaneous potential remains even at finite temperature.

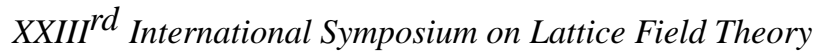

25-30 July 2005

Trinity College, Dublin, Ireland

\footnotetext{
* Speaker.
} 


\section{Introduction}

Recently, the color confinement scenario in Coulomb gauge has been attracting many scientists. Zwanziger [1] has discussed the color confinement mechanism in Coulomb gauge, which can be a physical gauge as compared with Lorentz-covariant gauge, and through the studies of the Coulomb gauge renormalization in Refs. [1, 2, 3], it has been reported that the time-time component of the gluon propagator $g^{2} D_{00}$, including the color-Coulomb instantaneous potential plus the non-instantaneous vacuum polarization, is invariant under the renormalization. (Here $g$ is a coupling constant of gauge field theory.) Furthermore, it has been pointed out in the recent work by Zwanziger [4], an inequality, $V_{\text {phys }}(R) \leq V_{\text {coul }}(R)$, where $V_{\text {phys }}(R)$ means the physical heavy quark potential and $V_{\text {coul }}(R)$ the color-Coulomb instantaneous potential. This inequality tells that if the physical potential is confining, then the Coulomb singlet potential is also confining. Indeed, in the $S U(2)$ numerical study by Cucchieri and Zwanziger [5], it has been found that the transverse gluon propagator $D^{\operatorname{tr}}(\vec{k})$ vanishes at $\vec{k}=0$, whereas the instantaneous color-Coulomb potential $D_{00}(\vec{k})$ is strongly enhanced at $\vec{k}=0$. Moreover, Greensite, Olejnik and Zwanziger have found in the $S U(2)$ lattice simulation that the color-Coulomb potential defined by the partial-length Polyakov line (PPL) correlator grows linearly at large distances [6, 7], which is deeply associated with the enhancement of $D_{00}$ at $\vec{k}=0$. The instantaneous part of $D_{00}$ may be managed with the PPL correlator.

In this work, we study the long-range behavior of heavy quark potential in Coulomb gauge in the quenched $S U$ (3) lattice QCD simulation with the PPL correlator. We investigate the behavior of the color-Coulomb potential in the confinement and deconfinement phases. The most interesting point is whether the color-Coulomb potential has a linearly rising feature at large quark separations. In the deconfinement phase, we will find that the linearity of the instantaneous part is not lost. In this report we will add the preliminary higher temperature results of the instantaneous part and give an argument of the relationship between the color-Coulomb string tensions and the magnetic scalings at finite temperature.

\section{Measurement}

A partial-length Polyakov line (PPL) is defined as [6, 7]

$$
L\left(\vec{x}, n_{t}\right)=\prod_{t=1}^{n_{t}} U_{0}(\vec{x}, t), \quad n_{t}=1,2, \cdots, N_{t} .
$$

Here $U_{0}(\vec{x}, t)=\exp \left(\operatorname{iag} A_{0}(\vec{x}, t)\right)$ is a $S U(3)$ link variable in the temporal direction and $a, g, A_{0}(\vec{x}, t)$ and $N_{t}$ mean a lattice cutoff, a gauge coupling, a time component gauge field and a temporal lattice size. A PPL correlator in the color $S U(3)$ singlet channel is given by

$$
G\left(R, n_{t}\right)=\frac{1}{3}\left\langle\operatorname{Tr}\left[L\left(R, n_{t}\right) L^{\dagger}\left(0, n_{t}\right)\right]\right\rangle .
$$

where $R$ is $|\vec{x}|$. One can obtain a potential on the lattice from them,

$$
V\left(R, n_{t}\right)=\log \left[\frac{G\left(R, n_{t}\right)}{G\left(R, n_{t}+1\right)}\right] .
$$


In particular, with the smallest temporal lattice extension $n_{t}=1$, we define

$$
V(R, 0)=-\log [G(R, 1)] .
$$

$V(R, 0)$ in Coulomb gauge corresponds to a color-Coulomb instantaneous potential, $V_{\text {coul }}(R)$. In the practical nonperturbative numerical study, the instantaneous contribution has been managed through $\mathrm{V}(\mathrm{R}, 0)$ [6, 7]; this also appears as the enhancement of $D_{00}$ at vanishing momentum [5]. The $V\left(R, n_{t}\right)$ at $n_{t} \rightarrow \infty$ further corresponds to a physical potential, $V_{p h y s}(R)$, which can be usually yielded from the Wilson loop calculation at $n_{t} \rightarrow \infty$. In addition, these two potentials obey Zwanziger's inequality, $V_{\text {phys }}(R) \leq V_{\text {coul }}(R)$ [4].

\section{Numerical Results}

We carry out the $S U(3)$ lattice gauge simulation at quenched level to calculate the colorCoulomb potential. The lattice configurations are generated by Heat-bath Monte-Carlo technique with a plaquette Wilson gauge action, and in order to fix the gauge we adopt the iterative method.

\subsection{Color-Coulomb $q \bar{q}$ potential}

The left-hand side of Fig. 1 shows the results of the color-Coulomb potential $V(R, 0)$, obtained by the PPL correlator with $n_{t}=1$. Those data are generated in the $18^{4}$ lattice simulation at $\beta=$ $5.85-6.00$. To obtain a string tension, we assume the following fitting function as a function of distance $R$,

$$
V\left(R, n_{t}\right)=C+K R+A / R, \quad A=-\pi / 12,
$$

where $C$ is a constant and $K$ corresponds to the string tension. ( The fittings work well; $\chi^{2} / n d f \sim$ $O(1)$ for the fitting range $R=2-6$.) It is found that the color-Coulomb potential $V(R, 0)$, rises linearly as the distance $R$ increases at $\beta=5.85-6.00$; it can be described with the linearly rising function. The string tensions for $\beta=5.85-6.00$ and the Wilson loop string tension [8] for $\beta=6.0$ are summarized in TABLE 1 . The color-Coulomb string tension at $\beta=6.0$ is approximately 2-3 times larger than the Wilson loop string tension.

Because the $V\left(R, n_{t}\right)$ at $n_{t} \rightarrow \infty$ corresponds to a physical potential, one expects that $V\left(R, n_{t}\right)$ as $\mathrm{T}$ increases becomes consistent with the Wilson loop potential $V_{w}(R)$. The $n_{t}$ dependence of the color-Coulomb potential at $\beta=6.0$ is displayed in the right-hand side of Fig. 1. (We use the $18^{3} \times 32$ lattice and 600 configurations measured by every 100 sweeps.) $V(R, T)$ may approach $V_{w}(R)$ as $T$ increases. The inequality, $V_{w(\text { phys })}(R) \leq V_{\text {coul }}(R)$ is also satisfied.

\subsection{Color-Coulomb potentials at finite temperature}

The finite temperature behavior of the color-Coulomb potential is shown in the left-hand side of Fig. 2. This simulation is carried out on the $24^{3} \times 6$ lattice at $\beta=6.11$, corresponding to $T / T_{c} \sim 1.50$, where $T$ stands for a system temperature and $T_{c}$ the critical temperature of the QGP phase transition. (The 300 configurations measured by every 100 steps are used here.) It is very interesting that even at finite temperature, $T / T_{c} \sim 1.50$, the color-Coulomb potential $V(R, 0)$, is surprisingly not screened and the linearly rising feature at large distances still exists. Fitting these data with the function Eq. (3.1), we obtain that the $K\left(\beta=6.11, T / T_{c} \sim 1.50\right)=0.118(1)$, and we 
Table 1: The fitted results of the string tensions. The $K$ and $\sigma$ are obtained from the singlet $V(R, 0)$, and the $K_{w}$ and $\sigma_{w}$ mean the Wilson loop string tension at $\beta=6.0$ [8], not calculated here. We use the relation $\sqrt{\sigma}=\sqrt{K} a^{-1}$ and the lattice cutoffs estimated from the Monte-Carlo renormalization analyses.

\begin{tabular}{lllll}
\hline$\beta$ & $K$ & $\sqrt{\sigma}[\mathrm{MeV}]$ & $K_{w}$ & $\sqrt{\sigma_{w}}[\mathrm{MeV}]$ \\
\hline \hline 5.85 & $0.2291(22)$ & $706(4)$ & & \\
5.90 & $0.1950(10)$ & $716(4)$ & & \\
5.95 & $0.1726(6)$ & $736(3)$ & & \\
6.00 & $0.1467(4)$ & $740(3)$ & $0.0513(25)[8]$ & $470(46)$ \\
\hline
\end{tabular}
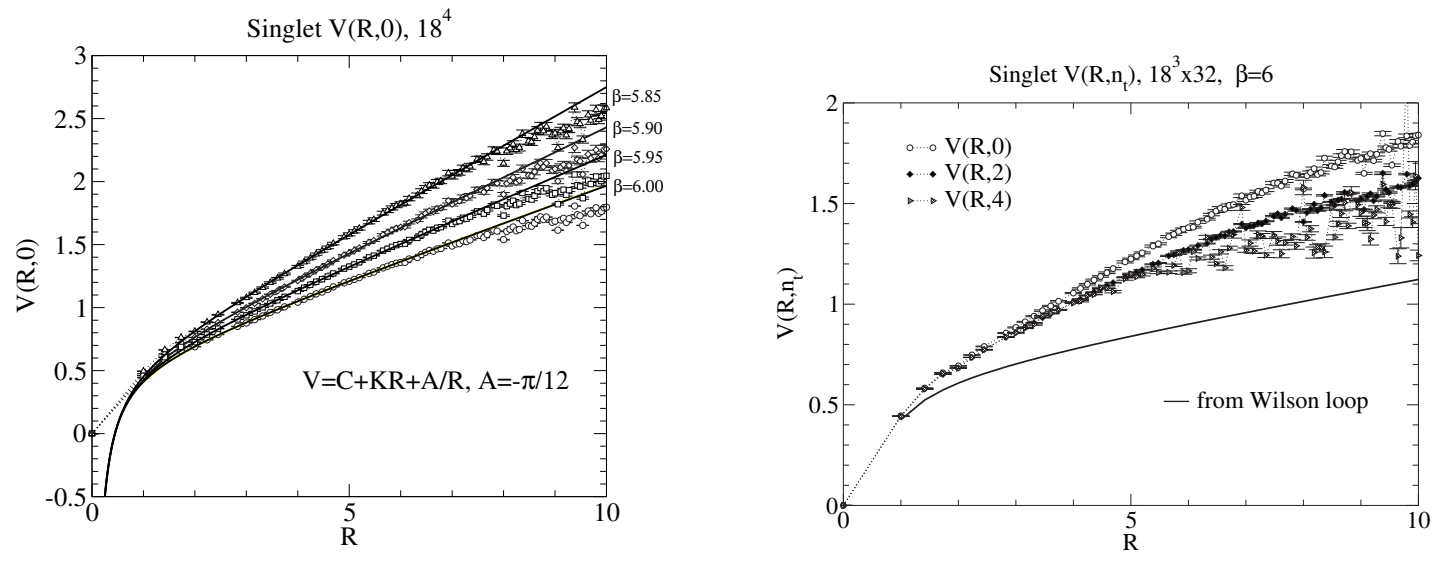

Figure 1: Behavior of the color-Coulomb instantaneous heavy $q \bar{q}$ potential in the confinement phase. Here $V\left(R, n_{t}\right)$ and $R$ have a non-dimensional lattice unit and for example the coupling constant $\beta=6.0$ corresponds to the lattice cutoff $a \sim 0.1 \mathrm{fm}$.

find that the $\sqrt{\sigma}\left(\beta=6.11, T / T_{c} \sim 1.50\right)=792(10) \mathrm{MeV}$, which is larger than the $\sqrt{\sigma}(\beta=6.0, T \sim$ $0)=740(4) \mathrm{MeV}$ listed in TABLE 1 . However, as the temporal extension $T$ increases, the $V\left(R, n_{t}\right) \mathrm{s}$ are screened at large distances, $R \gtrsim 1 / T=6$ on this lattice at least, and they are qualitatively consistent with the Polyakov line potential [9, 10].

Numerical results at higher temperature are also shown in the right-hand side of Fig. 2, (The 100 configurations measured by every 100 steps are used here.) We find that, even at $T / T_{c}=5.0$, the color-Coulomb instantaneous part is a linearly rising potential at large distances. In Fig. 3, the temperature dependence of the color-Coulomb string tensions at higher $T$ is shown and we did the fitting analysis using a magnetic scaling, $\sqrt{\sigma} / T \sim g^{2}(T)$ since the magnetic scaling dominates for the high temperature QCD. As a result, the color-Coulomb string tension at finite temperature may be described by the magnetic scaling.

\section{Concluding remarks}

We have studied the color-Coulomb potential defined with the partial-length Polyakov line correlator by the quenched $S U(3)$ lattice gauge simulation. Those $S U(3)$ results obtained in this 

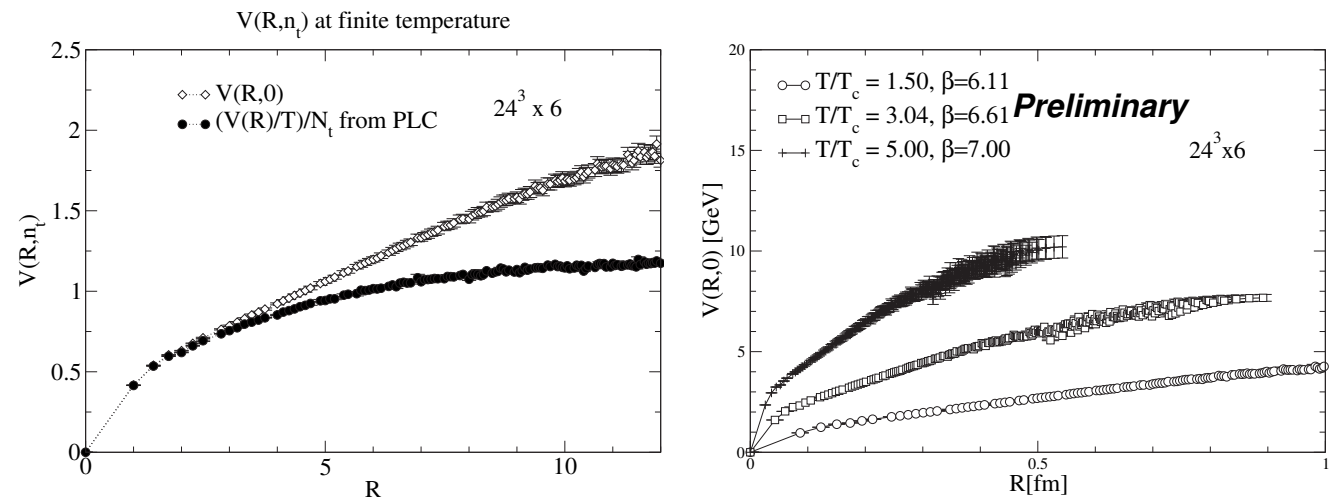

Figure 2: Behavior of the color-Coulomb instantaneous heavy $q \bar{q}$ potential in the deconfinement phase. In the graph of the left-hand side the non-dimensional lattice unit is used.

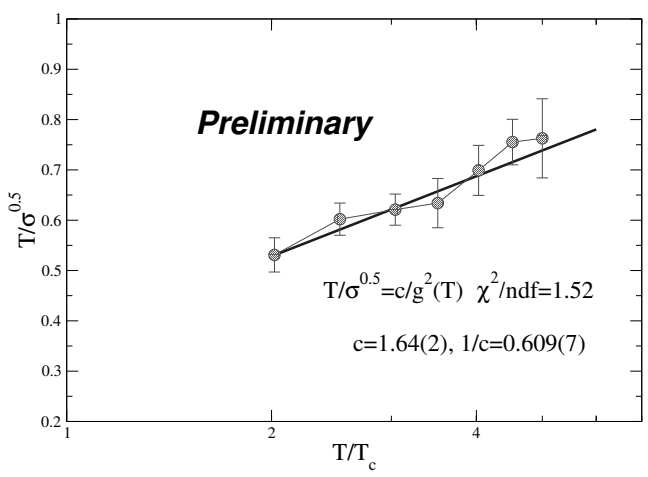

Figure 3: Temperature dependence of the color-Coulomb string tension in the deconfinement phase and the fitting analysis by the magnetic scaling. Here we assume $T / \sqrt{\sigma}=c / g^{2}(T)$, where $g(T)$ is a two-loop QCD running coupling constant.

study are qualitatively consistent with the $S U(2)$ analyses carried out by Greensite, Olejnik and Zwanziger [6, 7].

The color-Coulomb potential $V(R, 0)$ in the confinement phase grows linearly at large distances; its string tension is approximately several times larger than that of the usual Wilson loop potential. Moreover as the temporal extension $n_{t}$ of the PPL correlator increases, the color-Coulomb potential approaches asymptotically the usual Wilson loop potential.

On the other hand, the color-Coulomb potential $V(R, 0)$ in the QCD deconfinement phase at $T / T_{c} \sim 1.50$, is also the linearly rising function at large distances; namely, it is not screened. The color-Coulomb potential with the finite-temporal length in Coulomb gauge is screened sufficiently, and finally it becomes comparable with the screened potentials obtained from the full-temporal length Polyakov line correlator in Coulomb gauge.

It is important to use Coulomb gauge for the confinement scenario discussed in Refs. [1] and 
it is found that the color-Coulomb potential is a linearly confining potential by the $S U(3)$ lattice simulation used here as well as the $S U(2)$ lattice calculation [6, 7].

An extensive numerical study of the color-dependent force between two quarks may be significant to understand the color confinement and multiquark hadrons, although we here focus on the singlet channel for $q \bar{q}$. The other color-dependent forces in Coulomb gauge are also calculated and the results have been published in Ref. [11].

\section{Acknowledgment}

We would like to thank D. Zwanziger for much many helpful discussions. The calculation was done on SX-5(NEC) vector-parallel computer at RCNP of Osaka University. We really appreciate support of the RCNP administrators. This work is supported by Grant-in-Aid for Scientific Research by Monbu-Kagaku-sho (No.11440080, No. 12554008 and No. 13135216)

\section{References}

[1] D. Zwanziger, Nucl. Phys. B 518 (1998) 237-272.

[2] L. Baulieu, D. Zwanziger, Nucl.Phys. B548 (1999) 527-562, arXiv:hep-th/9807024.

[3] A. Cucchieri and D. Zwanziger, Phys. Rev. D65 (2002) 014002, arXiv:hep-th/0008248.

[4] D. Zwanziger, Phys. Rev. Lett. 90 (2003) 102001, arXiv:hep-lat/0209105.

[5] A. Cucchieri and D. Zwanziger, Phys. Rev. D65 (2002) 014001, arXiv:hep-lat/0008026.

[6] J. Greensite and Š. Olejník, Phys. Rev. D56, 094503 (2003), arXiv:hep-lat/0209068.

[7] J. Greensite, Š. Olejník, D. Zwanziger, Phys. Rev. D69, 074506(2004), arXiv:hep-lat/0401003.

[8] G. S. Bali, K. Schilling, Phys. Rev. D47 (1993) 661-672, arXiv:hep-lat/9208028.

[9] A. Nakamura and T. Saito, Prog.Theor.Phys. 112 (2004) 183-188, arXiv:hep-lat/0406038.

[10] A. Nakamura and T. Saito, Prog.Theor.Phys. 111 (2004) 733-743, arXiv:hep-lat/0404002.

[11] A. Nakamura and T. Saito, Phys. Lett. B621,(2005)171-175. 